\title{
Calculation of the Ettingshausen Coefficient in a Rectangular Quantum Wire with an Infinite Potential in the Presence of an Electromagnetic Wave (the Electron - Optical Phonon Interaction )
}

\author{
Cao Thi Vi Ba, Tran Hai Hung,, Doan Minh Quang, Nguyen Quang Bau \\ Faculty of Physics, VNU University of Science, 334 Nguyen Trai, Hanoi, Vietnam
}

Received 11 October 2017

Revised 24 October 2017; Accepted 25 October 2017

\begin{abstract}
The Ettingshausen coefficient (EC) in a Rectangular quantum wire with an infinite potential (RQWIP)in the presence of an Electromagnetic wave (EMW) is calculated by using a quantum kinetic equation for electrons. Considering the case of the electron - optical phonon interaction, we have found the expressions of the kinetic tensors $\sigma_{i k}, \beta_{i k}, \gamma_{i k}, \zeta_{i k}$. From the kinetic tensors, we have also obtained the analytical expression of the EC in the RQWIP in the presence of EMW as function of the frequency and the intensity of the EMW, of the temperature of system, of the magnetic field and of the characteristic parameters of RQWIP. The theoretical results for the EC are numerically evaluated, plotted and discussed for a specific RQWIP GaAs/GaAsAL. We also compared received $\mathrm{EC}$ with those for normal bulk semiconductors and quamtum wells to show the difference. The Ettingshausen effect in a RQWIP in the presence of an EMW is newly developed.
\end{abstract}

Keywords: Ettingshausen effect, Quantum kinetic equation, RQWIP, Electron - phonon interaction, kinetic tensor.

\section{Introduction}

Nowadays, the theoretical study of kinetic effects in low-dimensional systems is increasingly interested, especially on the electrical, magnetic and optical properties of the low-dimensional systems such as: the absorption of electromagnetic waves, the acoustomagnetoelectric effect, the Hall effect, ... These results show us that there are some significant differences from the bulk semiconductor that the previous researches studied [1-12]. Among those, the Ettingshausen effect has just been researched in bulk semiconductors [13] and only been studied on the theoretical basis in 2-D systems [14]. Furthermore, no research has been done on the Ettinghausen effect in 1-D systems such as quantum wires so far. In this paper, the calculation of Ettingshausen coefficient in the Rectangular quantum wire with an infinite

\footnotetext{
* Corresponding author. Tel.: 84-903293995.

Email: haihung307@gmail.com

https//doi.org/ 10.25073/2588-1124/vnumap.4236
} 
potential in the presence of magnetic field, electric field under the influence of electromagnetic wave is done by using the quantum kinetic equation method that brings the high accuracy and the high efficiency. Comparing the results obtained in this case with in the case of the bulk semiconductors and quantum wires, we see some differences. To demonstrate this, we estimate numerical values for a $\mathrm{GaAs} / \mathrm{GaAsAl}$ quantum wire.

\section{Calculation of the Ettingshausen coefficient in a Rectangular quantum wire with an infinite potential in the presence of an electromagnetic wave}

In a model, we consider a wire with rectangular cross section $\left(\mathrm{L}_{\mathrm{x}} \times \mathrm{L}_{\mathrm{y}}\right)$ and the length $L_{z}$. The effective mass of electron is denoted as $\mathrm{m}$. The RQWIP is subjected to a crossed dc electric field $\vec{E}_{1}=\left(0,0, E_{1}\right)$ and magnetic field $\vec{B}=(B, O, O)$ in the presence of a strong EMW characterized by electric field $\vec{E}(t)=\vec{E}_{0} \sin (\Omega t)$ (with $\mathrm{E}_{0}$ and $\Omega$ are the amplitude and the frequency of LR, respectively). Under these condition, the wave function and energy spectrum of confined electron can be written as:

$\psi_{\gamma, \vec{k}}(x, y, z)=\frac{1}{\sqrt{L_{z}}} e^{i \vec{k} z} \sqrt{\frac{2}{L_{x}}} \sin \left(\frac{n \pi x}{L_{x}}\right) \sqrt{\frac{2}{L_{y}}} \sin \left(\frac{l \pi y}{L_{y}}\right)$ when $\left\{\begin{array}{l}0 \leq x \leq L_{x} \\ 0 \leq y \leq L_{y}\end{array}\right.$

and $\psi_{\gamma, \vec{k}}(x, y, z)=0$ if else.

$$
\varepsilon_{\gamma}(k)=\frac{\hbar^{2} k_{z}^{2}}{2 m}+\frac{\pi^{2} \hbar^{2}}{2 m}\left(\frac{n^{2}}{L_{x}^{2}}+\frac{l^{2}}{L_{y}^{2}}\right)+\omega_{c}\left(N+\frac{1}{2}\right)-\frac{1}{2 m}\left(\frac{e E_{l}}{\omega_{c}}\right)^{2}
$$

where $\mathrm{k}_{\mathrm{z}}$ is the electron wave momentum; $\omega_{c}=\frac{e B}{m}$ is the cyclotron frequenciesn; $\gamma$ and $\gamma^{\prime}$ are the quantum numbers $(\mathrm{n}, \mathrm{l})$ and $\left(\mathrm{n}, \mathrm{l}^{\prime}\right)$ of electron; $\mathrm{N}, \mathrm{N}^{\prime}$ are the Landau level $(\mathrm{N}=0,1,2, \ldots)$. These expressions differ from the equivalent expressions in bulk semiconductors [14] and quantum wells [13].

The Hamiltonnian of the electron - optical phonon interaction system in the above RQWIP can be written as:

$$
\begin{aligned}
& H=\sum_{\gamma, \vec{k}} \varepsilon_{\gamma}\left(\vec{k}-\frac{e}{c} \vec{A}_{(t)}\right) a_{\gamma, \vec{k}}^{+} a_{\gamma, \vec{k}}+\sum_{\vec{q}} \omega_{\vec{q}} b_{\vec{q}}^{+} b_{\vec{q}}+ \\
& +\sum_{\gamma, \gamma^{\prime}, \vec{k}, \vec{q}}\left|C_{\vec{q}}\right|^{2}\left|I_{\gamma, \gamma^{\prime}}(\vec{q})\right|^{2} a_{\gamma, \vec{k}+\vec{q}}^{+} a_{\gamma^{\prime}, \vec{k}}\left(b_{\vec{q}}+b_{-\vec{q}}^{+}\right)+\sum_{\vec{q}} \varphi(\vec{q}) a_{\gamma, \vec{k}+\vec{q}}^{+} a_{\gamma^{\prime}, \vec{k}}
\end{aligned}
$$

Where $a_{\gamma, \vec{k}}^{+}$and $a_{\gamma, \vec{k}}\left(b_{\vec{q}}^{+}\right.$and $\left.b_{\vec{q}}\right)$ are the creation and the annihilation operators of electron (optical phonon); $\vec{k}$ is the electron wave momentum; $\vec{q}$ is the phonon wave vector; $\overrightarrow{\omega_{q}}$ are optical phonon frequency; $C_{\vec{q}}$ the electron - optical phonon interaction constant: $\left|C_{\vec{q}}\right|^{2}=\frac{e^{2} \omega_{o}}{2 \varepsilon_{0} q^{2} V}\left(\frac{1}{\chi_{\infty}}-\frac{1}{\chi_{0}}\right)$ (here $\mathrm{V}$ is the unit normalization volume, $\chi_{\infty}$ is magnetic permeability of high frequency dielectric, $\chi_{0}$ is magnetic permeability of static dielectric; $I_{\gamma, \gamma^{\prime}}(\vec{q})$ is the electron form factor, which is determinned by [8], different from that in cylindrical quantum wire; $\varphi(\vec{q})$ is the potential undirected: 
$\varphi(\vec{q})=(2 \pi i)^{3}\left(e \vec{E}+\omega_{c}[\vec{q}, \vec{h}]\right) \frac{\partial}{\partial \vec{q}} \delta(\vec{q})$

( $\vec{h}$ is unit vector in the direction of magnetic field).

Through some computation steps, the quantum kinetic equation takes the form:

$$
\begin{aligned}
& \frac{\sum_{\gamma, \vec{k}} \frac{e}{m} \vec{k} n_{\gamma, \vec{k}} \delta\left(\varepsilon-\varepsilon_{\gamma, \vec{k}}\right)}{\tau}+\omega_{c}\left[\vec{h}, \sum_{\gamma, \vec{k}} \frac{e}{m} \vec{k} n_{\gamma, \vec{k}} \delta\left(\varepsilon-\varepsilon_{\gamma, \vec{k}}\right)\right]= \\
& =-\frac{e}{m} \sum_{\gamma, \vec{k}} \vec{k}\left(\vec{F} \frac{\partial n_{\gamma, \vec{k}}}{\partial \vec{k}}\right) \delta\left(\varepsilon-\varepsilon_{\gamma, \vec{k}}\right)+\frac{2 \pi e}{m} \sum_{\gamma, \gamma^{\prime}, \vec{q}}\left|C_{\vec{q}}\right|^{2}\left|I_{\gamma, \gamma^{\prime}}(\vec{q})\right|^{2} N_{\vec{q}} \vec{k} \times \\
& \times\left\{[ \overline { n } _ { \gamma ^ { \prime } , \vec { q } + \vec { k } } - \overline { n } _ { \gamma , \vec { k } } ] \left[\left(1-\frac{\lambda^{2}}{2 \Omega^{2}}\right) \delta\left(\varepsilon_{\gamma^{\prime}, \vec{k}+\vec{q}}-\varepsilon_{\gamma, \vec{k}}-\omega_{o}\right)+\frac{\lambda^{2}}{4 \Omega^{2}} \delta\left(\varepsilon_{\gamma^{\prime}, \vec{k}+\vec{q}}-\varepsilon_{\gamma, \vec{k}}-\omega_{o}+\Omega\right)+\right.\right. \\
& \left.+\frac{\lambda^{2}}{4 \Omega^{2}} \delta\left(\varepsilon_{\gamma^{\prime}, \vec{k}+\vec{q}}-\varepsilon_{\gamma, \vec{k}}-\omega_{o}-\Omega\right)\right]+\left[\bar{n}_{\gamma^{\prime}, \vec{k}-\vec{q}}-\bar{n}_{\gamma, \vec{k}}\right]\left[\left(1-\frac{\lambda^{2}}{2 \Omega^{2}}\right) \delta\left(\varepsilon_{\gamma^{\prime}, \vec{k}-\vec{q}}-\varepsilon_{\gamma, \vec{k}}+\omega_{o}\right)+\right. \\
& \left.\left.+\frac{\lambda^{2}}{4 \Omega^{2}} \delta\left(\varepsilon_{\gamma^{\prime}, \vec{k}-\vec{q}}-\varepsilon_{\gamma, \vec{k}}+\omega_{o}-\Omega\right)+\frac{\lambda^{2}}{4 \Omega^{2}} \delta\left(\varepsilon_{\gamma^{\prime}, \vec{k}-\vec{q}}-\varepsilon_{\gamma, \vec{k}}+\omega_{o}+\Omega\right)\right]\right\} \delta\left(\varepsilon-\varepsilon_{\gamma, \vec{k}}\right)
\end{aligned}
$$

Equation (5) we put:

$$
\begin{aligned}
& \vec{R}(\varepsilon)=\sum_{\gamma, \vec{k}} \frac{e}{m} \vec{k}_{\gamma, \vec{k}} \delta\left(\varepsilon-\varepsilon_{\gamma, \vec{k}}\right) \\
& \vec{Q}(\varepsilon)=-\frac{e}{m} \sum_{\gamma, \vec{k}} \vec{k}\left(\vec{F} \frac{\partial n_{\gamma, \vec{k}}}{\partial \vec{k}}\right) \delta\left(\varepsilon-\varepsilon_{\gamma, \vec{k}}\right) ; \vec{F}=e \cdot \vec{E}_{1}-\frac{\varepsilon-\varepsilon_{F}}{T} \nabla T ; \\
& \vec{S}(\varepsilon)=\frac{2 \pi e}{m} \sum_{\gamma, \gamma^{\prime}, \vec{q}, \vec{k}}|C(\vec{q})|^{2}\left|I_{\gamma, \gamma^{\prime}}(\vec{q})\right|^{2} N_{\vec{q}} \vec{k} \times \\
& \times\left\{[ \overline { n } _ { \gamma ^ { \prime } , \vec { q } + \vec { k } } - \overline { n } _ { \gamma , \vec { k } } ] \left[\left(1-\frac{\lambda^{2}}{2 \Omega^{2}}\right) \delta\left(\varepsilon_{\gamma^{\prime}, \vec{k}+\vec{q}}-\varepsilon_{\gamma, \vec{k}}-\omega_{o}\right)+\frac{\lambda^{2}}{4 \Omega^{2}} \delta\left(\varepsilon_{\gamma^{\prime}, \vec{k}+\vec{q}}-\varepsilon_{\gamma, \vec{k}}-\omega_{o}+\Omega\right)+\right.\right. \\
& \left.+\frac{\lambda^{2}}{4 \Omega^{2}} \delta\left(\varepsilon_{\gamma^{\prime}, \vec{k}+\vec{q}}-\varepsilon_{\gamma, \vec{k}}-\omega_{o}-\Omega\right)\right]+\left[\bar{n}_{\gamma^{\prime}, \vec{k}-\vec{q}}-\bar{n}_{\gamma, \vec{k}}\right]\left[\left(1-\frac{\lambda^{2}}{2 \Omega^{2}}\right) \delta\left(\varepsilon_{\gamma^{\prime}, \vec{k}-\vec{q}}-\varepsilon_{\gamma, \vec{k}}+\omega_{o}\right)+\right. \\
& \left.\left.+\frac{\lambda^{2}}{4 \Omega^{2}} \delta\left(\varepsilon_{\gamma^{\prime}, \vec{k}-\vec{q}}-\varepsilon_{\gamma, \vec{k}}+\omega_{o}-\Omega\right)+\frac{\lambda^{2}}{4 \Omega^{2}} \delta\left(\varepsilon_{\gamma^{\prime}, \vec{k}-\vec{q}}-\varepsilon_{\gamma, \vec{k}}+\omega_{o}+\Omega\right)\right]\right\} \delta\left(\varepsilon-\varepsilon_{\gamma, \vec{k}}\right) .
\end{aligned}
$$

We obtain the following equations:

$$
\begin{aligned}
& \vec{R}(\varepsilon)=\frac{\tau(\varepsilon)}{1+\omega_{c}^{2} \tau^{2}(\varepsilon)}\left\{(\vec{Q}(\varepsilon)+\vec{S}(\varepsilon))-\omega_{c} \tau(\varepsilon)([\vec{h}, \vec{Q}(\varepsilon)]+[\vec{h}, \vec{S}(\varepsilon)])+\right. \\
& \left.+\omega_{c}^{2} \tau^{2}(\varepsilon)(\vec{Q}(\varepsilon)+\vec{S}(\varepsilon), \vec{h}) \vec{h}\right\} .
\end{aligned}
$$


After some approximate developing and computation steps, we obtain the expression of Ettinghausen coefficient as follows:

$$
P=\frac{1}{H} \frac{\sigma_{x x} \gamma_{x y}-\sigma_{x y} \gamma_{x x}}{\sigma_{x x}\left[\beta_{x x}^{T} \gamma_{x x}-\sigma_{x x}\left(\xi_{x x}^{T}-K_{L}\right)\right]}
$$

Here:

$$
\begin{aligned}
& \sigma_{x x}=\frac{e a \tau}{1+\omega_{c}^{2} \tau^{2}}+\frac{e b}{m}\left(1-\omega_{c}^{2} \tau^{2}\right) \frac{\tau^{2}}{\left(1+\omega_{c}^{2} \tau^{2}\right)^{2}} ; \sigma_{x y}=\frac{e a \tau}{1+\omega_{c}^{2} \tau^{2}} \cdot \omega_{c} \tau+\frac{e b}{m} \cdot \omega_{c} \tau \cdot \frac{\tau^{2}}{\left(1+\omega_{c}^{2} \tau^{2}\right)^{2}} \\
& \beta_{x x}=\frac{e \Omega b}{m T} \cdot\left(1-\omega_{c}^{2} \tau^{2}\right) \cdot \frac{\tau^{2}}{\left(1+\omega_{c}^{2} \tau^{2}\right)^{2}} \\
& \gamma_{x x}=\frac{\Omega b}{m} \cdot\left(1-\omega_{c}^{2} \tau^{2}\right) \cdot \frac{\tau^{2}}{\left(1+\omega_{c}^{2} \tau^{2}\right)^{2}} ; \gamma_{x y}=\frac{\Omega b}{m} \cdot \omega_{c} \tau \cdot \frac{\tau^{2}}{\left(1+\omega_{c}^{2} \tau^{2}\right)^{2}} \\
& \xi_{x x}^{T}=\frac{\Omega^{2} b}{m T} \cdot\left(1-\omega_{c}^{2} \tau^{2}\right) \cdot \frac{\tau^{2}}{\left(1+\omega_{c}^{2} \tau^{2}\right)^{2}} \\
& a=\frac{e \beta L_{x}}{4 m \sqrt{\pi}}\left(\frac{2 m}{\beta \hbar^{2}}\right)^{1 / 2} \exp \left\{\beta\left[\varepsilon_{F}+\frac{1}{2 m}\left(\frac{e E_{l}}{\omega_{c}}\right)^{2}-\frac{\pi^{2} \hbar^{2}}{2 m}\left(\frac{n^{2}}{L_{x}^{2}}+\frac{l^{2}}{L_{y}^{2}}\right)-\omega_{c}\left(N+\frac{l}{2}\right)\right]\right\} \\
& b=\frac{2 \pi e N_{o}}{m} \sum_{\gamma, \gamma^{\prime}}\left(A_{1}+A_{2}+A_{3}+A_{4}+A_{5}+A_{6}+A_{7}+A_{8}\right)\left(\frac{1}{\chi_{\infty}}-\frac{1}{\chi_{o}}\right) I . I_{\gamma, \gamma^{\prime}} e^{B} \\
& I=\exp \left(\beta\left[\varepsilon_{F}-\frac{\pi^{2} \hbar^{2}}{2 m}\left(\frac{n^{2}}{L_{x}^{2}}+\frac{l^{2}}{L_{y}^{2}}\right)-\omega_{c}\left(N+\frac{1}{2}\right)+\frac{1}{2 m}\left(\frac{e E}{\omega_{c}}\right)^{2}\right]\right), I_{\gamma, \gamma^{\prime}}=\int_{-\infty}^{+\infty}\left|I_{\gamma, \gamma^{\prime}}(\vec{q})\right|^{2} d \vec{q} \\
& A_{l}=\frac{\beta L_{x} k_{B} T e^{2}}{8 \sqrt{2} \pi^{3}} e^{-\beta \frac{B_{I I}}{2}}\left(\sqrt{\frac{\pi}{2 \beta m}} e^{-\beta \frac{B_{I I}}{2}}+\left(2 B_{I I} m\right)^{l / 2} K_{\frac{1}{2}}\left(\beta \frac{B_{11}}{2}\right)^{2}\right) \\
& A_{2}=-\frac{\beta L_{x} k_{B} T e^{4} E_{o}^{2} B_{I I} \sqrt{\pi}}{16 m^{2}(\beta / 8 m)^{3 / 2} \Omega^{4}} e^{-\beta \frac{B_{I I}}{2}}\left(\beta+\frac{1}{B_{I I}}\right) \\
& A_{3}=\frac{\beta L_{x} k_{B} T e^{4} E_{o}^{2} B_{13} \sqrt{\pi}}{16 m^{2}(\beta / 8 m)^{3 / 2} \Omega^{4}} e^{-\beta \frac{B_{13}}{2}}\left(\beta+\frac{1}{B_{13}}\right), A_{4}=\frac{\beta L_{x} k_{B} T e^{4} E_{o}^{2} B_{14} \sqrt{\pi}}{16 m^{2}(\beta / 8 m)^{3 / 2} \Omega^{4}} e^{-\beta \frac{B_{14}}{2}}\left(\beta+\frac{1}{B_{14}}\right) \\
& A_{5}=\frac{\beta L_{x} k_{B} T e^{2}}{8 \sqrt{2} \pi^{3}} e^{-\beta \frac{B_{15}}{4}}\left(\sqrt{\frac{\pi}{2 \beta m}} e^{-\beta \frac{B_{I 5}}{2}}+\left(2 B_{15} m\right)^{1 / 2} K_{\frac{1}{2}}\left(\beta \frac{B_{15}}{2}\right)^{2}\right) \\
& A_{6}=-\frac{\beta L_{x} k_{B} T e^{4} E_{o}^{2} B_{15} \sqrt{\pi}}{16 m^{2}(\beta / 8 m)^{3 / 2} \Omega^{4}} e^{-\beta \frac{B_{15}}{2}}\left(\beta+\frac{1}{B_{15}}\right)
\end{aligned}
$$




$$
\begin{aligned}
& A_{7}=\frac{\beta L_{x} k_{B} T e^{4} E_{o}^{2} B_{17} \sqrt{\pi}}{16 m^{2}(\beta / 8 m)^{3 / 2} \Omega^{4}} e^{-\beta \frac{B_{17}}{2}}\left(\beta+\frac{1}{B_{17}}\right), A_{8}=\frac{\beta L_{x} k_{B} T e^{4} E_{o}^{2} B_{18} \sqrt{\pi}}{2 m^{2}(\beta / 8 m)^{3 / 2} \Omega^{4}} e^{-\beta \frac{B_{I 8}}{2}}\left(\beta+\frac{1}{B_{18}}\right) \\
& B_{11}=\frac{\pi^{2} \hbar^{2}}{2 m}\left(\frac{n^{\prime 2}-n^{2}}{L_{x}^{2}}+\frac{l^{\prime 2}-l^{2}}{L_{y}^{2}}\right)+\omega_{c}\left(N^{\prime}-N\right)-\omega_{o}, \\
& B_{13}=B_{11}+\Omega, B_{14}=B_{11}-\Omega, B_{15}=B_{11}+2 \omega_{o}, B_{17}=B_{15}+\Omega, B_{18}=B_{15}-\Omega
\end{aligned}
$$

Here $\beta=1 /\left(k_{B} T\right) ; h_{x}=0, h_{y}=0, h_{z}=1 ; K_{L}, \tau, T, k_{B}, \chi_{0}, \chi_{\infty}, \varepsilon_{F}$ :is the lattice heat conductivity, the momentum laxation time, the temperature, the Boltzmann constant, the static dielecttric constant, the high frequency dielectric constant, and the Fermi level, respectively. The expressions of the kinetic tensors $\sigma_{i k}, \beta_{i k}, \gamma_{i k}, \zeta_{i k}(11-14)$ and of the EC (10) as well as functions of the frequency and the intensity of the EMW, of the temperature of system, of the magnetic field and of the characteristic parameters of RQWIP are different from those in bulk semiconductors and quamtum wells. It is newly developed in the quantum theory of Ettinghausen effect.

\section{Numerical results}

We will survey, plot and discuss the expressions for the case of a specific GaAs/GaAsAl quantum well. The parameters used in the calculations are as follows:

$$
\begin{aligned}
& \chi_{\infty}=10.9, \chi_{\infty}=12.9, \hbar \omega_{0}=36.25 \mathrm{meV}, \rho=5320 \mathrm{~kg} \cdot \mathrm{m}^{-3}, \Omega=3.10^{13} \mathrm{~s}^{-1}, \\
& \varepsilon_{F}=50 \mathrm{meV}, \tau=10^{-12} \mathrm{~s}, L_{x}=8.10^{-9} \mathrm{~m}, L_{y}=7.10^{-9} \mathrm{~m}, \mathrm{~m}=0,067 . \mathrm{m}_{0}\left(\mathrm{~m}_{0} \text { is the mass of a free electron }\right)
\end{aligned}
$$

In Fig. 1, we show the dependence of the EC on the laser frequency. From the figure, we see that the EC in RQWIP decreased is nonliner with the frequency, however, the EC in the quantum wells increased with the frequency [14]. This also demonstrates its difference in bulk semiconductors [13].

In Fig. 2, we show the dependence of the EC on laser amplitute. We found that the EC in RQWIP decreased is nonliner with laser amplitude. This is similar in the case of quantum wells, however, the EC in the quantum wire has decreased much faster than in quantum wells and in bulk semiconductors [13,14].

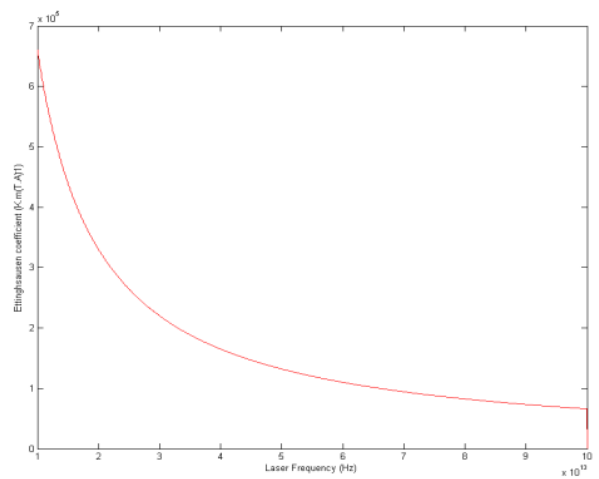

Fig 1. The dependence of EC on laser frequency.

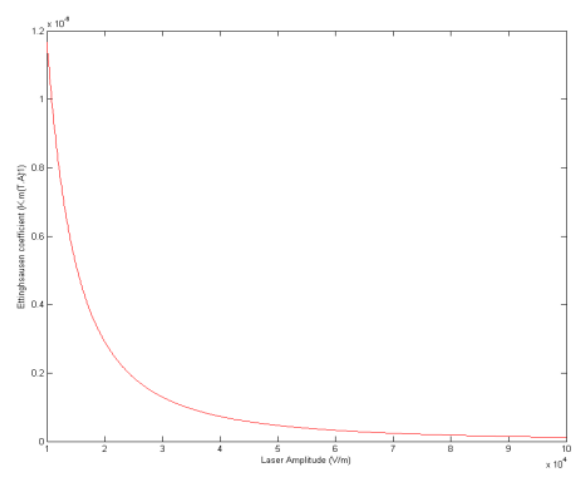

Fig 2. The dependence of EC on laser amplitute. 
In Fig. 3, we illustrate that the EC increase with the temperature T, however, the EC in the quantum wells decreased is nonliner with the frequency [14] and is different from bulk semiconductors [13].

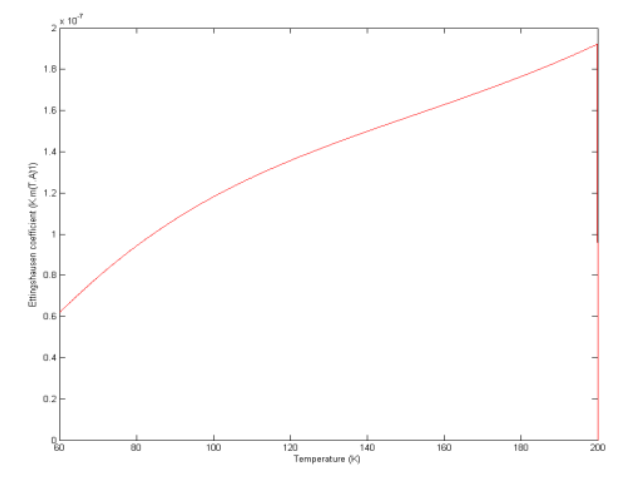

Fig 3. The dependence of EC on the temperature.

In Fig. 4, we show the dependence of the $\mathrm{EC}$ on $\mathrm{L}_{\mathrm{x}}, \mathrm{L}_{\mathrm{y}}$. It is the standard for us to evaluate the technology of making quantum wire, thereby choosing the best technology.
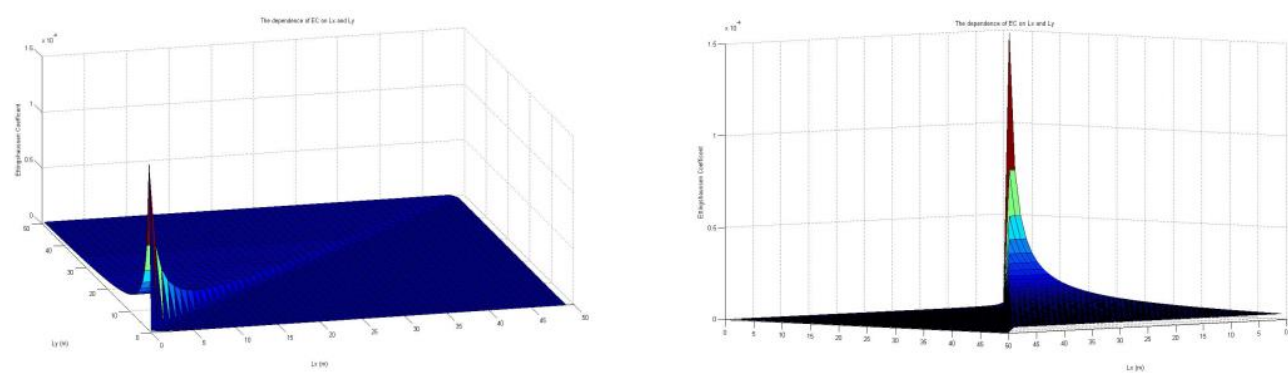

Fig 4. The dependence of EC on $\mathrm{L}_{\mathrm{x}}$ and $\mathrm{L}_{\mathrm{y}}$.

The above results show the difference between EC in quantum wires and in bulk semiconductors, in quantum wells. The cause is determined by material characteristics, expressed in wave function and energy spectrum.

\section{Conclusions}

In this paper, we researched Ettingshausen effect in a Rectangular quantum wire with an infinite potential in the presence of the magnetic. The electron - optical phonon interaction is taken into account at low temperatures, and the electron gas is nondegenerate. We obtain the analytical expression of Ettingshausen coeffection in a rectangular quantum wire. We see that the Ettingshausen coeffection in this case depend on some units such as: temperature, the amplitute of electromagnetic waves, the frequency of the radiation, phonon frequency and the parameters of a rectangular quantum wire. Estimating numerical 
values and graph for a GaAs/GaAsAl quantum wire to see clearly the nonlinear dependence of the Ettingshausen coeffection on the electromagnetic wave frequency. The more the electromagnetic wave amplitute and the temperature increase, the more the Ettingshausen coeffection decreases. However, Ettingshausen coeffecient reduced immediately if laser Amplitute increase. We also compared received EC with those for normal bulk semiconductors to show the difference.The Ettingshausen effect in a RQWIP in the presence of an EMW is newly developed.

\section{Acknowledgments}

This work is completed with financial support from the VNU (TN.17.06)

\section{References}

[1] Antonyuk V. B, MalŠ S. A. G, Larsson M. and Chao K. A. (2004). "Effect of electron-phonon interaction on electron conductance in one-dimensional systems". Phys. Rev. B, Vol. 69, pp. 155308-155314.

[2] N. Q. Bau, L. Dinh and T. C. Phong (2007). "Absorption coefficient of weak electromagnetic waves caused by confined electrons in quantum wires". Journal of the Korean Physical Society, Vol. 51, pp. 1325-1330.

[3] N. Q. Bau and B. D. Hoi (2012). 'Influence of a strong electromagnetic wave (laser radiation) on the Hall effect in quantum wells with a parabolic potential". Journal of the Korean Physical Society, Vol. 60, No. 1, pp. 59 - 64 (ISI).

[4] N. Q. Bau, N. V. Hieu and N. V. Nhan (2012). "The quantum acoustomagnetoelectric field in a quantum well with a parabolic potential”. Superlattices and Microstructure, Vol. 52, pp. 921-930.

[5] N. Q. Bau and H. D. Trien (2010). "The nonlinear absorption of a strong electromagnetic wave by confined electrons in rectangular quantum wires". PIERS Proceedings, Xi'an, China, pp. 336-341.

[6] Bennett R., Guven K., and Tanatar B. (1998). "Confined-phonon effects in the bandgap renormalization of semiconductor quantum wires". Phys. Rev. B, Vol. 57, pp. 3994- 3999.

[7] Brandes T. and Kawabata A. (1996). "Conductance increase by electron-phonon interaction in quantum wires". Phys. Rev. B, Vol. 54, pp. 4444-4447.

[8] Kim K.W., Stroscio M. A., Bhatt A., Mickevicius R. and Mitin V. V. (1991). "Electron-optical-phonon scattering rates in a rectangular semiconductor quantum wire". J. Appl. Phys., Vol. 70, pp. 319-327.

[9] Yu. S. G, K. W. Kim, M. A. Stroscio, G. J. Iafrate and A. Ballato(1996). "Electron interaction with confined acoustic phonons in cylindrical quantum wires via deformation potential". J. Korean Phys, Vol. 80, 2815.

[10] N. Q. Bau, N. T. Huong (2016). "Dependence of the Hall Coefficient on a length of rectangular quantum wires with infinitely high potential under the influence of a laser Radiation", Journal of Physics Conference Series, Vol. 726, pp. $012014-012019$.

[11] N. Q. Bau and N. T. Huong (2016). "The Hall Coefficient and Magnetoresistance in rectangular quantum wires with infinite potential under the influence of a Laser Radiation". International Journal of Physical and Mathematical Sciences - World Academy of Science, Engineering and Technology, Vol.10 (3), pp 75-80.

[12] Bau. N. Q., Hung. D. M., and Hung. L. T. (2010). "The influences of confined phonons on the nonlinear absorption coefficient of a strong electromagnetic wave by confined electrons in doping superlattices". PIER Letter 15, pp. 175-185.

[13] B.V.Paranjape and J.S.Levinger (1960). "Theory of the Ettingshausen Effect in Semiconductors". Phys. Rev., Vol.120, pp 437-451.

[14] D. T. Hang, D. T. Ha, D. T. T. Duong, N. Q. Bau (2016). "The Ettingshausen coefficient in quantum wells under the influence of laser radiation in the case of electron-optical phonon interaction". Photonics Letters of Poland, Vol 8(No 3), pp 79-81. 